\section{Cahiers de Narratologie}

Analyse et théorie narratives

$11 \mid 2004$

Figures de la lecture et du lecteur

\title{
Les adresses au lecteur chez Balzac
}

\section{Aude Deruelle}

\section{OpenEdition}

\section{Journals}

Édition électronique

URL : http://journals.openedition.org/narratologie/11

DOI : 10.4000/narratologie. 11

ISSN : 1765-307X

Éditeur

LIRCES

\section{Référence électronique}

Aude Deruelle, «Les adresses au lecteur chez Balzac », Cahiers de Narratologie [En ligne], 11 | 2004, mis en ligne le 01 janvier 2004, consulté le 10 décembre 2020. URL : http://journals.openedition.org/ narratologie/11 ; DOI : https://doi.org/10.4000/narratologie.11

Ce document a été généré automatiquement le 10 décembre 2020.

\section{(c) $(1) \&$}

Cahiers de Narratologie - Analyse et théorie narratives est mis à disposition selon les termes de la licence Creative Commons Attribution - Pas d'Utilisation Commerciale - Pas de Modification 4.0 International. 


\title{
Les adresses au lecteur chez Balzac
}

\author{
Aude Deruelle
}

Je ne vous ferai pas l'injure de vous l'expliquer ;
vous avez trop de bon sens et d'instruction pour
cela !...
Grand Dieu! quel lèse-lecteur je commettrais !1

Les romans de Balzac contiennent de nombreuses adresses au lecteur, au point qu'ils ont pu être traités par Zola de "véritable causerie avec le public $»^{2}$. Ce trait frappant de l'écriture balzacienne a suscité diverses études ${ }^{3}$, mais son originalité n'a peut-être pas été suffisamment mise en lumière. En effet, si le texte balzacien formule fréquemment des adresses à son destinataire, aucune apostrophe ne comporte les expressions «ami lecteur", "cher lecteur», ou tout simplement «lecteur». Et lorsque le narrateur s'adresse à son narrataire en employant la troisième personne, la présence du mot «lecteur» est exceptionnelle. L'ensemble de La Comédie humaine contient vingt-huit occurrences ${ }^{4} \mathrm{du}$ terme " lecteur » appliqué au narrataire : c'est très peu, compte tenu de l'ampleur de l'œuvre. Comment interpréter la quasi-absence de ce terme, alors même que le procédé de l'adresse au lecteur est extrêmement fréquent? De quoi témoigne un tel refus? Celui-ci, loin d'être fortuit ou anecdotique, mène au cœur des enjeux de l'adresse balzacienne au lecteur. Seront prises en compte les adresses qui empruntent la forme de l'apostrophe, ainsi que les discours adressés au lecteur au moyen de la troisième personne - les références implicites à un destinataire étant laissées de côté.

2 Quelques-unes des occurrences du mot « lecteur » sont intéressantes, car elles suscitent un métadiscours qui les commente. Il s'agit de l'adresse « au lecteur » ${ }^{5}$ qui ouvre L'Elixir de longue vie, seule œuvre de La Comédie humaine qui débute ainsi. Si les autres récits sont souvent dédiés à des connaissances de Balzac, ici l'adresse ouvrant la nouvelle joue le rôle d'une dédicace au lecteur : «La lecture nous donne des amis inconnus, et quel ami qu'un lecteur! nous avons des amis connus qui ne lisent rien de nous! l'auteur espère avoir payé sa dette en dédiant cette œuvre diis ignotis ». Dans cette dédicace, Balzac rappelle les circonstances qui l'ont conduit à écrire la nouvelle, avant de préciser :

Si l'auteur a conservé cette vieille formule AU LECTEUR, dans un ouvrage où il tâche de représenter toutes les formes littéraires, c'est pour placer une remarque relative 
à quelques Études, et surtout à celle-ci. Chacune de ses compositions est basée sur des idées plus ou moins neuves, dont l'expression lui semble utile, il peut tenir à la priorité de certaines formes, de certaines pensées qui, depuis, ont passé dans le domaine littéraire, et s'y sont parfois vulgarisées. Les dates de la publication primitive de chaque Étude ne doivent donc pas être indifférentes à ceux des lecteurs qui voudront lui rendre justice ${ }^{6}$.

Il est nécessaire de resituer la dédicace dans son contexte. L'Elixir de longue vie est un petit récit publié en 1830. L'adresse « au lecteur» qui ouvre le livre a été ajoutée en 1846, à l'occasion de la réédition de la nouvelle dans l'édition «Furne ». Or cette nouvelle relève d'une écriture dite " frénétique ", à la mode en 1830, qui reposait tant sur des procédés mélodramatiques que sur des images terrifiantes. La fin du récit illustre bien cette mode littéraire. Le héros, don Juan, ressuscite grâce à un merveilleux élixir, mais seule sa tête a bénéficié de cette résurrection :

Alors cette tête vivante se détacha violemment du corps qui ne vivait plus et tomba sur le crâne jaune de l'officiant.

«Souviens-toi de dona Elvire », cria la tête en dévorant celle de l'abbé.

Ce dernier jeta un cri affreux qui troubla la cérémonie. Tous les prêtres accoururent et entourèrent leur souverain.

«Imbécile, dis donc qu'il y a un Dieu? » cria la voix au moment où l'abbé, mordu

dans sa cervelle, allait expirer ${ }^{7}$.

Il s'agit là d'une prose fort éloignée de l'esthétique romanesque à l'œuvre dans La Comédie humaine, et ce malgré les différentes couleurs que peut prendre la palette de Balzac. L'auteur use d'une "vieille formule ", l'adresse au lecteur, pour justifier l'insertion d'une nouvelle vieillie, et ce malgré ses dénégations, puisqu'il suggère habilement que ses innovations d'alors ont été vulgarisées depuis.

5 Cette occurrence apporte donc un premier élément de réponse : l'adresse " au lecteur » (du moins en envoi au début du récit) est inscrite dans une tradition littéraire galvaudée. On comprend que d'ordinaire Balzac refuse d'employer cette "vielle formule », alors même qu'il tente d'instaurer une nouvelle écriture romanesque.

6 Cette tradition est celle des romans parodiques et anti-romans (Le Roman comique de Scarron, Le Roman bourgeois de Furetière, les œuvres de Fielding, Tristram Shandy de Sterne par exemple) : lorsque l'on parle, à propos du récit romanesque, d'adresse au lecteur, le modèle qui se présente à l'esprit est celui de l'interpellation à la Diderot. Inversement, $L a$ Princesse de Clèves, œuvre sérieuse, ne contient pas d'adresse au destinataire, comme si ce procédé était lié à un discours de type parodique. À cet égard, Balzac apparaît doublement novateur. Tout en écrivant un roman sérieux ${ }^{8}$, il a recours à l'adresse au lecteur: "ses récits ne sont, sauf exceptions, ni critiques ni parodiques, il fourmillent pourtant de marques de destinataire $»^{9}$.

7 Les adresses au lecteur des romans parodiques peuvent varier dans leurs formulations (Scarron vouvoie son lecteur, Furetière le tutoie dans sa préface et le vouvoie dans son récit), mais une caractéristique frappante est la fréquence du terme même de «lecteur ", utilisé soit dans une interpellation, soit dans un discours qui s'adresse au lecteur à la $3^{\mathrm{e}}$ personne. Ce terme peut figurer seul ou être assorti de qualificatifs: "cher lecteur", " ami lecteur», "lecteur bénévole». Voici, par exemple, l'envoi de Furetière : «Ami lecteur, quoique tu n'achètes et ne lises ce livre que pour ton plaisir, si néanmoins tu n'y trouvais autre chose, tu devrais avoir regret à ton temps et à ton argent $»^{10}$. Ces adresses au lecteur ont majoritairement lieu quand le texte refuse de se soumettre à des topoï 
romanesques (ce que le lecteur est en droit d'attendre de la lecture d'un roman), ou quand il n'applique pas des règles élémentaires de composition :

Je me doute bien qu'il n'y aura pas un lecteur (tant soit-il bénévole) qui ne dise ici en lui-même : « Voici un méchant Romaniste ! Cette histoire n'est pas fort longue ni fort intriguée. Comment ! il conclut d'abord un mariage, et on n'a coutume de les faire qu'à la fin du dixième tome $! »^{11}$.

Si vous vous attendez, Lecteur, que ce livre soit la suite du premier, et qu'il y ait une connexité nécessaire entre eux, vous êtes pris pour dupe $\mathrm{e}^{12}$.

L'hypothèse suivante peut donc être émise : si le terme « lecteur » est absent des adresses balzaciennes au destinataire, c'est parce qu'il constitue une évocation trop manifeste du procédé anti-romanesque de l'adresse au lecteur ${ }^{13}$ : or le récit balzacien ne vise pas à dénoncer l'artificialité des intrigues et des topoï romanesques, mais repose sur un souci de vraisemblance qui structure l'histoire.

Ce refus du terme " lecteur ", et plus globalement de l'adresse parodique au destinataire, est d'autant plus frappant que ce procédé perdure à l'époque de Balzac dans les récits excentriques ${ }^{14}$ de "petits romantiques " comme Nodier ou Gautier. Voici comment Gautier s'adresse à son lecteur :

Puisque ce Fortunio tant désiré n'est pas encore arrivé, et que sans lui nous ne pouvons commencer notre histoire, nous demanderons au lecteur la permission de lui esquisser les portraits des compagnes de Musidora, à peu près comme on remet un livre d'images ou un album plein de croquis à quelqu'un qu'on est obligé de faire attendre ${ }^{15}$.

L'adresse au lecteur, associée ici à un autre procédé, la métalepse, s'inscrit dans la tradition éprouvée du roman parodique.

Plus précisément, ce refus de la tradition parodique et excentrique est un choix poétique lié à l'esthétique romanesque mise en œuvre dans La Comédie humaine ${ }^{16}$. Deux faits viennent en effet l'attester. Tout d'abord, dans ses romans de jeunesse, Balzac emploie principalement l'adresse au lecteur traditionnelle. Il y a ainsi une soixantaine d'occurrences du terme « lecteur » appliqué au destinataire dans le seul Jean-Louis - soit plus du double des cas figurant dans l'ensemble de La Comédie humaine! En voici quelques exemples :

Ici, lecteur, j'ai un compte à régler avec vous ; quoique je n'aie pas tant de mémoire que vous, je me souviens fort bien que j'ai le droit de mettre dans ce susdit ouvrage deux cents et quelques pages dont la substance équivaille à rien.

Lecteurs, ne vous impatientez pas de ma manière de raconter, chacun a la sienne. Mon cher lecteur, connaissez-vous la rue Saint-Germain-l'Auxerrois? Certainement. - Eh bien, elle aboutit au Grand Châtelet. - Je le sais. - En ce cas, nous coïncidons dans nos vues ${ }^{17}$.

Interpellations au singulier ou au pluriel, adresse assortie du qualificatif traditionnel "cher", ainsi que dialogue avec le lecteur tout à fait dans la tradition sternienne: si Balzac maîtrisait le procédé de l'adresse parodique, il y renonce dans les romans de $L a$ Comédie humaine, où aucune adresse de ce type ne figure - ce qui prouve qu'il s'agit là d'un choix d'ordre poétique ${ }^{18}$.

En outre, neuf des vingt-huit occurrences de La Comédie humaine figurent dans la Physiologie du mariage. Certaines se présentent même sous la forme de l'apostrophe, alors que ce procédé est banni dans le reste de l'œuvre balzacienne : «Disciples de Panurge, de vous seuls je veux pour lecteurs "; « c'est à vous-même, vous, lecteur " ${ }^{19}$. Balzac recourt ici à une adresse dans la tradition parodique, comme en témoigne aussi cette apostrophe 
qui figure dans la conclusion : «Homme de Dieu qui m'as suivi jusqu'ici !... j'espère qu'une petite récapitulation ne t'effraiera pas, et j'ai voyagé dans la conviction que tu te disais comme moi : "Où diable allons-nous ?" " ${ }^{20} \mathrm{Ce}$ cas ne constitue pourtant pas un démenti de notre hypothèse : en effet, non seulement la Physiologie du mariage est une œuvre des débuts (1826, remaniée en 1829), mais elle ne ressortit pas à une écriture romanesque : cette physiologie se présente sous la forme d'un discours principalement argumentatif.

Ainsi, le refus du terme "lecteur» manifeste un choix poétique: une interpellation rappelant les adresses parodiques, dans le cadre du projet romanesque de La Comédie humaine, n'est plus envisageable. D'ailleurs, les rares fois où le texte balzacien a recours à ce terme de «lecteur ", le propos fait entendre une tonalité parodique, et ce même si le récit, dans son ensemble, repose sur une énonciation sérieuse: "Il est nécessaire d'expliquer ici, car tous les lecteurs n'ont pas eu la fantaisie de visiter un bagne, que chaque forçat [...] »; «Si, dans le nombre, assez restreint, des lecteurs qui s'occupent de la partie morale ou philosophique d'un livre $[. ..]{ }^{21}{ }^{21}$. Le deuxième exemple, notamment, recèle une attaque assez virulente qui rappelle la manière dont le narrateur parodique peut malmener son destinataire.

(s) narrataire, comment s'adresse-t-il à lui ? Ce qui frappe au premier abord, c'est la très grande variété des moyens servant à désigner le narrataire: loin d'opter pour l'appellation unique de «lecteur », le texte balzacien multiplie les désignations. Deux cas sont possibles.

Premièrement, quand il veut s'adresser à l'ensemble de ses lecteurs, Balzac privilégie le recours aux pronoms. Au sein de formules qui s'adressent au lecteur à la troisième personne, on trouve les pronoms indéfinis « on » ou " chacun »: «Mais on va voir que tout homme instruit [...]», "Chacun devine que la façade [...] $»^{22}$. Dans le cadre des interpellations, le lecteur est désigné par l'intermédiaire d'un « vous »: «Un des traits qui le rendirent célèbre suffira sans doute pour vous expliquer M. Hochon tout entier $»^{23}$. Balzac a également recours à des impératifs telles que «Imaginez », « Figurez-vous », lors de portraits ou de descriptions de paysage. L'ouverture de La Grenadière constitue une exploitation remarquable de ce procédé : «En entrant, vous trouverez un petit palier où commence un escalier tortueux ", "La Loire est à vos pieds. Vous la dominez d'une terrasse élevée de trente toises au-dessus de ses eaux capricieuses; le soir vous respirez ses brises venues fraîches de la mer " ", "N’offrez pas de prix! La Grenadière ne sera jamais à vendre $»^{24}$. Ce procédé est certes un moyen rhétorique pour rendre la description plus vivante. Mais il permet aussi de créer le décor fictionnel par un geste pour le moins original : le narrateur anime son narrataire, lui prêtant les attributs d'un personnage, pour poser les lieux de l'action. Ce type d'adresse reposant sur des pronoms permet ne pas dessiner une image particulière du destinataire, tout en le désignant précisément : en ce sens, on peut les voir comme des substituts du terme de "lecteur ", trop connoté.

Dans le deuxième cas, très fréquent, le narrateur s'adresse à une catégorie précise de narrataires. On trouve par exemple l'expression «ceux qui »: "Ceux qui s'amusent à étudier la nature sociale $»^{25}$, ou des périphrases, comme dans ce passage des Chouans: «les personnes pour lesquelles ces tableaux ne sont pas sans mérite $[. ..] »^{26}$. Enfin, le narrateur peut choisir de s'adresser à une catégorie particulière du public (les femmes, les bourgeois, les Parisiens...).

Ce procédé de la catégorisation est lié à la mise en place d'un pacte de lecture pédagogique. En se faisant roman du savoir, le récit balzacien se hisse au-dessus du pur 
divertissement. Ce savoir apparait notamment dans des digressions (sur l'architecture, l'histoire, les sciences occultes, etc.) qui sont la marque du texte balzacien. Lors de ces séquences digressives qui tournent à la leçon, le narrateur adopte la posture du savant et place le lecteur dans la position d'un élève ignorant. Cependant, il s'agit de faire endosser au destinataire ce rôle sans l'accabler sous le poids de cette accusation: le lecteur pourrait refuser le pacte pédagogique et « sauter » la séquence, voire refermer le livre. La solution consiste alors à mettre en évidence les lacunes d'une catégorie de lecteurs - le narrateur se voyant dans la nécessité de pallier ce manque de connaissance :

Cette explication est nécessaire, autant pour éviter les sottes critiques de ceux qui ne savent rien que pour constater les grandes choses d'un monde qui, dit-on, s'en va, et que tant de gens poussent sans le comprendre ${ }^{27}$.

Ici, si l'attaque est violente, la cible reste floue. L'usage de la relative déterminative « ceux qui ne savent rien » permet de délimiter une catégorie de lecteurs tout en ne la désignant pas explicitement, les ignorants étant uniquement caractérisés par leur manque de savoir. L'adresse au lecteur entre alors dans une stratégie énonciative visant à faire accepter le pacte pédagogique.

20 Le narrateur peut néanmoins délimiter de manière plus explicite la catégorie des narrataires ignorants, au moyen de divers critères. Les critères géographiques ont souvent été pointés : l'opposition Paris / province est bien connue chez Balzac ${ }^{28}$. En voici un exemple : «Personne hors Paris ne sait, et personne à Paris n'ignore qu'un juge au tribunal de commerce est le plus étrange magistrat qu'une Société se soit permis de créer ${ }^{29}$. Selon les occurrences, ce sont les provinciaux ou les Parisiens qui sont taxés d'ignorance.

21 L'opposition géographique peut être différente, lorsque le narrateur convoque les étrangers, à qui il est nécessaire de peindre les us et coutumes de la France.

Il est peu de flâneurs qui n'aient rencontré cette geôle roulante; mais, quoique la plupart des livres soient écrits uniquement pour les Parisiens, les étrangers seront sans doute satisfaits de trouver ici la description de ce formidable appareil de notre justice criminelle ${ }^{30}$.

Peut-être suffira-t-il de peindre la division de $\mathrm{M}$. de la Billiardière, pour que les étrangers et les gens qui vivent en province aient des idées exactes sur les mœurs intimes des bureaux, car ces traits principaux sont sans doute communs à toutes les administrations européennes ${ }^{31}$.

Le premier exemple érige le roman en document qui renseigne sur les mœurs d'un pays : Splendeurs et misères des courtisanes, contre-guide touristique, décrit les envers du monde parisien, ici le moyen de locomotion très particulier qu'est le panier à salade. Mais le plus frappant est la dissociation entre deux catégories de narrataires. Cet exemple met en évidence le caractère stratégique de la catégorisation : tout en reconnaissant la primauté du lectorat parisien, le narrateur ne le prend pas en compte pour pouvoir faire une digression, les «étrangers » faisant figure de boucs émissaires aisés à mettre en cause ce qui pourrait se gloser par un « je sais que vous savez, mais il faut penser aux autres ». Dans le deuxième exemple, les provinciaux sont mis sur le même plan que les étrangers. Ce parallèle, que l'on retrouve à plusieurs reprises, peu flatteur pour les provinciaux qui se voient en quelque sorte exclus de la France, a surtout une dimension stratégique: mieux vaut désigner plusieurs cibles afin de légitimer la nécessité du discours savant.

23 Fait qui a été moins souligné, le clivage peut s'établir au moyen de critères sociaux, annulant la dichotomie Paris / province : «Sans ce petit détail, une honnête bourgeoise ne comprendrait pas comment une fortune se fond entre les mains de ces créatures ${ }^{32}$. 
Cette formulation peut paraître péjorative, car elle met en évidence un esprit borné par une morale de classe, mais elle a également pour but d'affrioler le destinataire. Le narrateur propose en effet à ses lectrices d'aborder les rivages inconnus du monde des filles, de ces « créatures » - terme évidemment utilisé par ces bourgeoises pour désigner les courtisanes, et dont l'emploi a ici une valeur polyphonique. Un autre exemple de catégorisation sociale repose sur le même principe :

$\mathrm{Au}$ commencement de cette Scène, il est nécessaire d'expliquer, une fois pour toutes, aux gens habitués à la moralité des familles bourgeoises, que les paysans n'ont, en fait de mœurs domestiques, aucune délicatesse ${ }^{33}$.

Il ne s'agit rien de moins que d'aller explorer le territoire inconnu de ces sauvages, les paysans, qui n'ont aucune morale, tout particulièrement en ce qui concerne les « mœurs domestiques", terme pudique, mais non obscur, pour désigner la sexualité. En stigmatisant l'ignorance d'une partie des narrataires, le narrateur attise donc la curiosité de tout lecteur.

Un autre type de clivage, établi selon des critères chronologiques, dévoile plus particulièrement la dimension stratégique du procédé :

Ce ne sera certes pas un hors-d'œuvre que de décrire ce coin de Paris actuel, plus tard on ne pourrait pas l'imaginer; et nos neveux, qui verront sans doute le Louvre achevé, se refuseraient à croire qu'une pareille barbarie ait subsisté pendant trentesix ans, au cœur de Paris ${ }^{34}$.

[...] pour faire comprendre entièrement à des lecteurs du dix-neuvième siècle comment des événements assez vulgaires en apparence étaient devenus surnaturels, et pour leur faire partager les frayeurs du vieux temps, il est nécessaire d'interrompre cette histoire pour jeter un rapide coup d'œil sur les aventures de maître Cornélius ${ }^{35}$.

Dans le premier exemple, il s'agit d'instruire les générations à venir de lecteurs, qui ignoreront les coutumes du XIX ${ }^{e}$ siècle. Cette solution est très consensuelle, aucun lecteur vivant du temps de Balzac ne pouvant se sentir visé dans son manque de savoir. Une telle stratégie permet également l'érection d'un monument, La Comédie humaine, destiné à être le témoignage de toute une époque. Inversement, dans le deuxième exemple, ce sont de fait tous les lecteurs contemporains de Balzac dont l'ignorance est pointée par le texte. Il s'agit donc d'une fausse catégorisation ${ }^{36}$.

Lorsque le narrateur s'adresse à une classe de narrataires, et non à l'ensemble de ses lecteurs, l'adresse sérieuse n'est pas incompatible avec une certaine ironie. Les lecteurs accusés d'ignorance peuvent offrir matière à raillerie. Dans Les Petits Bourgeois, on peut lire cette adresse: «Les étrangers, les gens de province, les gens du monde ne savent peut-être pas que les avocats sont aux avoués ce que sont les généraux aux maréchaux $»^{37}$. On retrouve les clivages géographiques classiques (les "étrangers", les "gens de province »), mais vient s'y adjoindre un troisième terme pour le moins surprenant, établi quant à lui selon des critères sociaux. Si cette dernière catégorie de lecteur ignore la différence entre l'avoué et l'avocat, ce n'est pas parce qu'elle n'habite pas Paris, mais parce qu'elle n'a pas souvent affaire directement à la justice. Quoique cette explication soit tout à fait respectueuse envers la classe sociale ainsi désignée, la juxtaposition a une valeur clairement ironique.

Ces différents exemples auront montré la variété des catégorisations de narrataire mises en place dans le texte balzacien. De telles catégorisations dessinent a contrario l'image d'un narrateur non inscrit dans des limites géographiques, des sphères sociales, voire des périodes historiques: la fameuse omniscience du narrateur balzacien ne se comprend 
donc que par rapport aux connaissances incomplètes de narrataires épars. Ces catégorisations des narrataires permettent de faire la lumière sur le lien qu'entretient le texte balzacien avec son lecteur.

vs

Le refus du terme «lecteur » et le choix d'un autre type de désignation ne manifestent pas seulement le désir de mettre à distance la tradition parodique afin de créer une écriture romanesque originale. Ils expriment également le rejet d'un certain rapport avec le destinataire, que privilégiait cette tradition, mais que l'on trouve également dans des romans sérieux contemporains de l'écriture balzacienne.

Des adresses parodiques découle un rapport ambigu avec le lecteur. Ce qui prime, c'est incontestablement la supériorité du premier sur le second. Dans Tristram Shandy de Sterne, le narrateur annonce qu'il va consacrer cinquante pages au siège de Calais avant de renoncer à ce projet : «Courage, cher lecteur ! Je méprise le procédé ; il me suffit de te tenir en mon pouvoir, mais profiter de l'avantage que je dois à la fortune de la plume c'en serait trop $! \aleph^{38}$ Le narrateur affiche sa toute-puissance en déconstruisant son intrigue et en brisant l'illusion romanesque (c'est le fameux « il ne tiendrait qu'à moi » de Jacques le fataliste) : "Vous voyez, lecteur, combien je suis obligeant; il ne tiendrait qu'à moi de donner un coup de fouet aux chevaux $\|^{39}$. Quant au narrataire, il n'est pas seulement support d'un discours critique à l'encontre des topoï romanesques, il est souvent la cible de ce discours :

Lecteurs et spectateurs indolents tirent un grand avantage de ces prologues ou chapitres liminaires : comme ils ne sont pas obligés de voir les uns ou de lire les autres et que la pièce ou le livre sont ainsi retardés, les premiers leur donnent un quart d'heure de plus à rester à table et les seconds leur permettent de commencer leur lecture à la quatrième ou cinquième page au lieu de la première $[. . .]^{40}$.

31 Cependant, souvent moqué, le lecteur n'en est pas moins toujours pris en compte, et de façon explicite. Un dialogue constant se tisse tout au long de la narration de l'histoire, qui engendre une familiarité entre destinateur et destinataire. Ces quelques lignes de Fielding, à la fin de Tom Jones, traduisent bien cette relation :
Adieu au lecteur
Et maintenant, lecteur, nous voici arrivés à la dernière étape de notre long voyage. Ayant donc parcouru ensemble tant de pages, comportons-nous l'un envers l'autre comme des compagnons de voyage qui ont passé plusieurs jours ensemble dans une diligence, et qui, en dépit de toutes les petites piques ou animosités qui ont pu se produire au long de la route, se réconcilient en fin de compte et grimpent pour la dernière fois dans le véhicule avec gaieté et bonne humeur; puisque aussi bien, après cette étape, il peut nous arriver, comme c'est généralement le cas pour eux, de ne plus jamais nous revoir ${ }^{41}$.

Or l'emploi récurrent du terme même de «lecteur » contribue fortement à l'instauration de ce rapport. En effet, la familiarité n'est pas seulement celle du ton, qui traite le lecteur d'" ami ", ou de "cher " (même si ces expressions paraissent parfois ironiques), mais provient surtout du commerce régulier entre deux instances stables: le narrateur, désigné dans son acte même de produire le discours, et le narrataire, figure fixe érigée et modelée au fil du récit par le retour de cette unique appellation.

Ce type de rapport instauré avec le lecteur, fondé sur la familiarité, est aussi celui privilégié par certains auteurs contemporains de Balzac, et dont les œuvres ne s'inscrivent pourtant pas dans la tradition parodique. C'est le cas des romans de Stendhal, et de Notre-Dame de Paris de Hugo (1831) - et ce même si ces écritures romanesques sont 
par ailleurs fondamentalement distinctes l'une de l'autre. Voici certains exemples stendhaliens :

[...] en faveur du lecteur, comme disent les gens qui crient le discours du roi à l'ouverture de la session, nous allons donner quelques passages du dialogue des deux vieux généraux ${ }^{42}$.

Les salons que ces messieurs traversèrent au premier étage, avant d'arriver au cabinet du marquis, vous eussent semblé, ô mon lecteur, aussi tristes que magnifiques. On vous les donnerait tels qu'ils sont, que vous refuseriez de les habiter ; c'est la patrie du bâillement et du raisonnement triste ${ }^{43}$.

Chez Stendhal, comme on le voit, les adresses au lecteur ne sont pas dénuées d'ironie, et rappellent le procédé parodique, quoique inscrites dans un récit sérieux. Plus systématiquement encore, dans Notre-Dame de Paris, on relève de nombreuses adresses contenant le terme de « lecteur » qui rythment le récit :

Le lecteur n'a peut-être pas oublié l'effronté mendiant [...].

Nous sommes ravis d'avoir à apprendre à nos lecteurs que pendant toute cette scène $[. .$.$] .$

Cette procession, que nos lecteurs ont vue partir du Palais [...].

Vous avez été enfant, lecteur, et vous êtes peut-être assez heureux pour l'être encore ? $^{44}$

Ces adresses hugoliennes façonnent au fil du récit une figure du « lecteur », avec laquelle s'instaure le rapport de familiarité.

Or Balzac ne cherche pas à instaurer ce type de rapport. En effet, au sein d'un même récit, le narrateur peut s'adresser successivement aux Parisiens, aux bourgeois, aux provinciaux, aux femmes, aux hommes... Ces catégorisations, suscitées par les séquences dans lesquelles elles prennent place, constituent autant de stratégies ponctuelles, mais elles contribuent également à la labilité du destinataire. Il est vrai que certaines œuvres suscitent de façon privilégiée des narrataires précis. Les Scènes de la vie parisienne et les Scènes de la vie provinciale auront respectivement pour narrataire ignorant le provincial et le parisien, une Scène de la vie privée s'adressera plus particulièrement aux femmes, etc. Mais l'instabilité fondamentale du narrataire balzacien se retrouve à l'échelle supérieure qu'est l'ensemble de La Comédie humaine. Dans un roman, et d'un roman à l'autre, la position du narrataire est occupée par des catégories différentes : « les exclus peuvent se trouver inclus, et vice versa $»^{45}$. Cette instabilité de la figure du narrataire est d'autant plus frappante qu'elle contraste avec la permanence du narrateur balzacien, dont les postures sont similaires tout au long du récit, d'un récit à l'autre, et à ce titre aisément reconnaissables par le lecteur aguerri.

La mise en évidence de ce principe d'instabilité du narrataire permet de mieux mesurer ce qui, en ce qui concerne l'adresse au lecteur, sépare le texte balzacien des romans parodiques de Diderot, et des œuvres du jeune Balzac, où c'est bien toujours le même narrataire qui est interpellé et qui accompagne le narrateur tout au long du récit. Dans les romans de La Comédie humaine, aucune familiarité n'est possible avec ce narrataire marqué par l'instabilité : les catégorisations successives empêchent le destinataire de se fixer en une image unique avec laquelle le dialogue pourrait se poursuivre.

En outre, ce principe d'instabilité permet de revenir sur le débat de l'autoritarisme du texte balzacien. On a souvent parlé de la coercition qu'il exerce sur son lecteur par l'entremise d'un narrataire soumis, élève docile qui lit les leçons de son maître. Selon Jean Rousset, "le lecteur se voit confirmer sa position naturelle de dépendance $»^{46}$. Les impératifs, notamment, ont suscité de telles analyses, comme manifestation la plus 
évidente d'un texte contraignant. La supériorité du narrateur balzacien sur le narrataire diffère cependant de l'autorité affectée par le narrateur du roman parodique. En effet, cette hiérarchie ne réside pas en la toute-puissance d'un narrateur libre de mener à sa guise son récit, mais en la détention d'un savoir encyclopédique.

À l'encontre de cette interprétation du fonctionnement du texte balzacien, on peut mettre en évidence que le narrateur tisse parfois de vrais liens de complicité avec son narrataire, le traitant non en inférieur, mais en égal. L'incipit de Ferragus dessine ainsi une connivence entre narrateur et destinataires «amants de Paris »: «Ces observations, incomprises au-delà de Paris, seront sans doute saisies par ces hommes d'étude et de pensée, de poésie et de plaisir qui savent récolter, en flânant dans Paris, la masse de jouissances flottantes $»^{47}$. Le lecteur parisien est invité à accéder à une lecture fondée sur la complicité. Reste que seule une catégorie de narrataires se voit ici accorder ce statut privilégié.

Pour contester la vision d'un texte balzacien purement autoritaire, on a pu également montrer à juste titre que le lecteur réel est invité à se démarquer par rapport au narrataire, disqualifié dans son manque de savoir ou de sensibilité - Franc Schuerewegen a analysé en ce sens le début du Père Goriot, qui conduit le lecteur à prendre des distances avec un narrataire rétif ${ }^{48}$. Stigmatiser une partie des narrataires permet de nouer des relations privilégiées avec les lecteurs qui ne sont pas accusés d'ignorance : à côté du rapport d'autorité et d'enseignement, se tisse un rapport de complicité avec les lecteurs.

41 Surtout, les multiples catégorisations de lecteurs, loin de dessiner une image stable d'un destinataire toujours soumis à l'autorité du narrateur, vont à l'encontre d'un rapport purement hiérarchique. Le lecteur (réel) doit sans cesse se positionner par rapport à ce narrataire mouvant: il s'identifie à un narrataire pour mieux se démarquer quelques pages plus loin d'un autre narrataire, et s'il accède à la position de complice du narrateur il peut se voir rétrograder peu après à la position d'élève ignorant. Une telle instabilité remet en question la vision d'un texte purement coercitif : le texte balzacien serait bien plus autoritaire s'il s'adressait à un lecteur unique car figé, comme dans le roman parodique. L'étoilement des narrataires compense donc ce que peut avoir de contraignant le pacte pédagogique. Et si « le lecteur balzacien n'est pas figé, fixé une fois pour toutes » ${ }^{49}$, c'est bien parce que le texte balzacien refuse le terme de "lecteur", qui aurait l'inconvénient d'unifier sous une même appellation des narrataires que le narrateur du texte balzacien se complaît, au contraire, à différencier.

Les adresses balzaciennes au lecteur peuvent certes être vues comme une trace d'oralité manifestant que le roman ne s'est pas encore extrait, en cette première moitié du XIXe siècle, de son passé de conte. Mais ce procédé joue de fait un rôle important dans la poétique balzacienne. Il s'agit pour l'auteur de La Comédie humaine de légitimer le roman en luttant contre les critiques qui pèsent sur ce genre littéraire, jugé trop futile et décrié dans sa propension à n'être qu'une affabulation. C'est ainsi que le récit balzacien se fait roman du savoir, en délivrant des connaissances à son destinataire. En changeant les enjeux du genre romanesque, le texte balzacien est amené à modifier l'horizon d'attente du lecteur: "prévoir son Lecteur Modèle ne signifie pas uniquement "espérer" qu'il existe, cela signifie aussi agir sur le texte de façon à le construire $»^{50}$. Les adresses au lecteur visent notamment à faire accepter au destinataire cette nouvelle esthétique romanesque. Dans la deuxième moitié du dix-neuvième siècle, lorsque les acquis de cette poétique auront donné une légitimité au roman en tant que genre, ces adresses ne s'imposeront plus comme nécessaires : les œuvres de Flaubert ou de Zola, dénuées de ce 
procédé, visent au contraire à l'effacement de la situation d'interlocution - de là peutêtre le jugement de l'auteur des Rougon-Macquart.

\section{NOTES}

1. BALZAC, Jean-Louis (1822), in Premiers Romans, Paris, Robert Laffont, « Bouquins », 1999, p. 415.

2. Cité par David BAGULEY, «Balzac, Zola, et la paternité du naturalisme », ", in Balzac. Une poétique du roman, sous la direction de Stéphane Vachon, Saint-Denis, PUV, 1996, p. 392.

3. Voir Jean ROUSSET, "L'inscription du lecteur dans La Comédie humaine », in Le Lecteur intime : de Balzac au journal, Paris, José Corti, 1986 ; Franc SCHUEREWEGEN, « Réflexions sur le narrataire », Poétique, Paris, Éditions du Seuil, n 70, 1987, p. 247-254. ; Éric BORDAS, Balzac, discours et détours. Pour une stylistique de l'énonciation romanesque, Toulouse, Presses universitaires du Mirail, 1997, p. 243-276.

4. Recherche effectuée sur le cédérom Explorerla Comédie humaine, Acamedia, 1999. Les occurrences figurant dans les préfaces ne sont pas comptabilisées : rappelons que cellesci ne figurent plus dans l'édition Furne. Par ailleurs, les préfaces instaurent un rapport entre auteur et lecteur différent de celui que le narrateur balzacien établit avec son destinataire.

5. BALZAC, L'Elixir de longue vie, Paris, Gallimard, « Bibliothèque de la Pléiade ", sous la direction de Pierre-Georges Castex, 1976-1981, XI, p. 473. Les autres citations de Balzac renverront sauf avis à cette édition.

6. Ibid., p. 474.

7. Ibid., p. 495.

8. Voir Erich AUERBACH, Mimésis. La Représentation de la réalité dans la littérature occidentale (1946), Paris, Gallimard, « Tel », 1968, 1994, p. 477.

9. Jean ROUSSET, op. cit., p. 38.

10. FURETIÈRE, Le Roman bourgeois (1666), Gallimard, « Folio », 1981, p. 23.

11. Ibid., p. 44.

12. Ibid., p. 167.

13. Albert Savarus contient une nouvelle écrite par le personnage éponyme. Dans l'édition originale, l'un des chapitres de cette nouvelle s'intitule « Une réflexion plus ou moins agréable au lecteur ». Il est frappant de constater que ce titre, contenant une adresse avec le terme de « lecteur » qui rappelle la littérature parodique, s’inscrit dans un récit marqué au coin de nombreux clichés romantiques, comme si Balzac avait à cœur de condamner la pratique galvaudée du procédé en le rattachant à une écriture romanesque peu originale.

14. Daniel SANGSUE, Le Récit excentrique, Paris, José Corti, 1987, p. 98.

15. GAUTIER, Fortunio (1837), in CEuvres, Paris, Robert Laffont, « Bouquins », 1995, p. 485.

16. Certes, le titre de La Comédie humaine ne date que de 1842. Mais dès Les Chouans (1829) figurent certains traits propres à l'écriture romanesque balzacienne (descriptions, discours explicatifs notamment). 
17. BALZAC, Jean-Louis , op. cit., p. 410, p. 470, p. 293.

18. Notons également les emplois ludiques de l'adresse au lecteur qui figurent dans Les Contes drolatiques - en revanche, le terme « lecteur » n'est pas utilisé. Après la description d'un quartier de Tours, le narrateur interpelle ainsi son destinataire : «Y estes-vous ? Oui. Bon. Adonques, la fille avoyt là sa buanderie [...] » (in Euvres diverses, I, p. 392). Cette écriture ludique ne contamine pas la rédaction des Études de mœurs à laquelle se consacre Balzac à la même période.

19. BALZAC, Physiologie du mariage, XI, p. 917 et p. 956.

20. Ibid., p. 1196.

21. BALZAC, Splendeurs et misères des courtisanes, VI, p. 839 et p. 642.

22. BALZAC, Les Paysans, IX, p. 257, p. 258.

23. BALZAC, La Rabouilleuse, IV, p. 419.

24. BALZAC, La Grenadière, II, p. 422-425.

25. BALZAC, Les Paysans, IX, p. 273.

26. BALZAC, Les Chouans, VIII, p. 913.

27. BALZAC, Les Secrets de la princesse de Cadignan, VI, p. 350.

28. Voir Jean ROUSSET, op. cit., p. 39

29. BALZAC, César Birotteau, VI, p. 272.

30. BALZAC, Splendeurs et misères des courtisanes, VI, p. 697. Nous soulignons.

31. BALZAC, Les Employés, VII, p. 957. Nous soulignons.

32. BALZAC, Splendeurs et misères des courtisanes, VI, p. 617.

33. BALZAC, Les Paysans, IX, p. 91.

34. BALZAC, La Cousine Bette, VII, p. 99.

35. BALZAC, Maître Cornélius, XI, p. 29.

36. Voir également cette catégorisation arithmétique : « Les neuf dixièmes des lecteurs et les neuf dixièmes du dernier dixième ignorent certainement les différences considérables qui séparent ces mots : Inculpé, Prévenu, Accusé, Détenu [...] (Splendeurs et misères des courtisanes, VI, p. 699). Le procédé est alors ironique (on remarque d'ailleurs la présence du terme « lecteur ») : la répétition de l'expression « neuf dixièmes », réduisant à peau de chagrin le nombre de lecteurs ignorants, dévoile que cette catégorisation est une manière de ne pas dire « tous les lecteurs ».

37. BALZAC, Les Petits Bourgeois, VIII, p. 154.

38. STERNE, Tristram Shandy (1767), Paris, Garnier, « GF », 1982, p. 438.

39. DIDEROT, Jacques le fataliste (1780), Paris, Gallimard, « Folio », 1973, p. 97.

40. FIELDING, Tom Jones (1749), Paris, Galllimard, « Folio »,1964, t. II, p. 322.

41. Ibid., p. 409

42. STENDHAL, Lucien Leuwen (1838, date d'écriture), Paris, Gallimard, « Folio », p. 85.

43. STENDHAL, Le Rouge et le Noir (1830), Paris, Gallimard, « Folio », p. 285.

44. HUGO, Notre-Dame de Paris (1831), Paris, Robert Laffont, « Bouquins », 1985, p. 530, p. 530, p. 541 , p. 562.

45. Jean ROUSSET, op. cit. p. 40.

46. Ibid., p. 43.

47. BALZAC, Ferragus, V, p. 794-795.

48. Franc SCHUEREWEGEN, art. cit., p. 249.

49. Christèle COULEAU, "La voix de son maître ", in La Voix narrative, textes réunis par Jean-Louis Brau, Publication de la Faculté des Lettres de Nice, 2001, t. I, p. 329

50. Umberto ECO, Lector in fabula, Paris, Grasset, « Le Livre de Poche », 1985, p. 69. 
INDEX

Index chronologique : XIXe siècle

\section{AUTEUR}

AUDE DERUELLE

Université de Nice-Sophia Antipolis, CIRCPLES, EA 3159 